\section{P-237 UNDERSTANDING THE NEED FOR RESPITE CARE AT DOVE HOUSE HOSPICE: A RETROSPECTIVE AUDIT}

George Obita, Anna Wolkowski, Catherine Bristow, John Creasey, Rachael Dixon, Nic Macmanus, Sarah Cash. Dove House Hospice, Hull, UK

\subsection{6/bmjspcare-2017-hospice.262}

Background UK policy and guidance frequently refers to respite care as a key factor in supporting carers. (NICE, 2004; Department of Health, 2005) However, little is known about respite care in palliative and end of life care and the role of hospices in providing respite care. (Wolkowski \& Carr, 2017; Vandepitte et al., 2016; Wolkowski et al., 2010). Dove House Hospice (DHH) in Hull provides respite care. Our observation is that the needs of both the patient and carer are frequently complex and patients are often highly dependent, with a high level of nursing and sometimes medical needs.

Aims To increase our understanding of the characteristics of respite care patients and carers with a view to articulating the need for hospice respite care, its benefits and potential benefits.

Methods A retrospective audit of records of respite care patients (2015-2016) was carried out. A simple data collection tool, organised on a Microsoft Excel spread sheet was used to extract and analyse data. Data collected included demographic and patient characteristics, diagnosis and condition background, referral/admission details, living arrangements and outcome of admission.

Results The majority of the 90 patients admitted for respite care were male (58\%). Complex neurological disorders were the leading background diagnosis (28\%) among the 16 diagnosis groups identified. Most referrals were from the community services or from patients and their families. Although most patients, $80 \%$, were discharged home after respite, about $12 \%$ died during the course of the respite. As this was a retrospective audit, we could not examine quality of care or impact of the intervention on patients or carers because the information was not available.

Conclusion The audit has enhanced our understanding of respite care patients and their carers. Results from this audit will provide a platform for further enquiry helping to guide and improve service provision.

\section{P-238 STAFF NURSE ROTATION INPATIENT TO COMMUNITY - BREAKING THE BARRIERS TO CARE SETTINGS}

Helen Reeves, Katie Taroni. St Giles Hospice, Whittington, UK

\subsection{6/bmjspcare-2017-hospice.263}

Background Palliative care teams have historically worked in isolation from other services. St Giles has revolutionised their workforce by incorporating staff nurse rotation between community and palliative care services.

Aim To provide seamless patient care, St Giles Hospice has incorporated rotation of staff nurses between community and inpatient palliative care services. The aim of this is to streamline patient journeys between services, increase staff knowledge between departments, maintain clinical skills and offer career development for staff.

Methods Staff nurses between departments were identified to be part of the pilot rotation, timeframes were set for rotation with evaluations at the beginning and end to evaluate the pilot and the benefits it had had. Patient feedback was also evaluated for any reference to the rotational role.

Results The initial rotation timeframe was too short, feedback from the nurses was that six months in each department would be more efficient and enable embedding into practice. Clinical skills in the community have been maintained with nurses able to perform more clinical tasks (bloods, medications etc) with increased confidence and competence. In addition the handover of care to inpatient settings has been improved with patients having a familiar face when they enter an unfamiliar setting. Due to the success this has now been rolled out to all staff nurses in the community with recruitment underway for their rotational counterparts.

Conclusion To date the rotation has been a success. We now have a rotation of CNS and Sister between departments and also rotation between staff nurses as well. As patients do not see barriers between services neither should staff. A greater understanding of each department enables better outcomes for patients and also staff with greater appreciation of roles.

\section{P-239 THE INSIDE OUT HOSPICE PROJECT - ARDGOWAN HOSPICE}

Joyce Sweeney, Ian Marshall, Ian Wilson. Ardgowan Hospice, Greenock, UK

\subsection{6/bmjspcare-2017-hospice.264}

Introduction Ardgowan Hospice has delivered specialist palliative care services to the people of Inverclyde for the past 35 years. Potential recipients of the services have had to travel to our hospice buildings, this coupled with the need for professional referral has limited the range of people who could benefit from much needed support. Traditionally we have offered support through in-patient stays, day services including therapy, bereavement, family and child support along with home care services. This model has been unchanged since the hospice opened.

Aims With the support of the Big Lottery we will transform the services that are offered to the people of Inverclyde. To make them more accessible, more inclusive and reaching deeper and wider into communities, in essence being able to offer our support where, when and how they want, services delivered.

Method Through a consultation across communities and in collaboration with partners we have an understanding of the current view of what the hospice provides and the view of how care should be delivered in the future. This engagement was via online survey and hardcopy format distributed across Inverclyde. 21 focus groups and six open groups were held where participants were guided by the questionnaire in an open discussion.

Results Response to the survey was $41 \%$. Over 500 comments were received over the course of the consultation from both individual responses and group settings. The analysis of the needs and wishes is now being carried out, this will determine how we will transform the services to meet the needs.

Conclusion On completion of the analysis Ardgowan Hospice will work collaboratively with communities and partners to develop appropriate responses and implement the necessary changes to achieve the project goals, reviewing delivery across the four year term to ensure that services continue to meet needs. 